\title{
MICROMECHANICAL MODELING OF BRITTLE FRACTURE OF FRENCH RPV STEEL. A COMPREHENSIVE STUDY OF STRESS TRIAXIALITY EFFECT.
}

\author{
Jean-Philippe MATHIEU \\ Material and Mechanics of Components Department \\ EDF R\&D - Les Renardières F-77818 Moret-sur-Loing \\ Email: jean-philippe.mathieu@edf.fr - Phone: +33160737427

\section{Karim INAL} \\ Ecole des Mines de Saint Etienne - CMP G. Charpak Gardanne \\ Email: inal@emse.fr
}

\author{
Olivier DIARD \\ EDF - CNPE de Penly \\ Email: olivier.diard@edf.fr
}

\section{Sophie BERVEILLER}

ENSAM CER de Metz - LPMM Metz

Email: sophie.berveiller@metz.ensam.fr

\begin{abstract}
The present study describes a multiscale representation of mechanisms involved in brittle fracture of a french Reactor Pressure Vessel (RPV) steel (16MND5 equ. ASTM A508 Cl.3) at low temperatures. Attention will be focused on the representation of stress heterogeneities inside the ferritic matrix during plastic straining, which is considered as critical for further micromechanical approach of brittle fracture. This representation is tuned on experimental results [1].

Modeling involves micromechanical a description of plastic glide, a mean field (MF) model and a realistic three-dimensional aggregates Finite Element (FE) simulation, all put together inside a multiscale approach. Calibration is done on macroscopic stress-strain curves at different low temperatures, and modeling reproduces experimental stress heterogeneities.

This modeling allows to apply a local micromechanical fracture criterion of crystallographic cleavage for triaxial loadings on the Representative Volume Element (RVE). Deterministic computations of time to fracture for different carbide sizes random selection provide a probability of fracture for an Elementary Volume (EV) consistant with the local approach.

Results are in good agreement with hypothesis made by local approach to fracture. Hence, the main difference is that no
\end{abstract}

phenomenological dependence on loading or microstructure is supposed for probability of fracture on the EV : this dependence is naturally introduced by the micromechanical description.

\section{NOMENCLATURE}

\begin{tabular}{|c|c|c|c|}
\hline 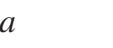 & : scalar quantity. & $\underset{\sim}{\mathbf{A}}$ & $2^{\text {nd }}$ order tensor. \\
\hline & : vector. & $\underset{\approx}{\mathbf{A}}$ & $4^{\text {th }}$ order tensor. \\
\hline $\operatorname{sign}(a)$ : & $: \quad \frac{a}{|a|}$. & & $\frac{\int_{V} a \cdot d V}{V}$ \\
\hline
\end{tabular}

\section{INTRODUCTION}

Brittle fracture of steels can be regarded from both microscopic and macroscopic aspects. At the microscopic scale, brittle fracture of low-alloyed steel is usually interpreted as intragranular cleavage [2]. Micromechanisms of fracture are still discussed, but a certain consensus can be established about the essential role of plasticity and presence of micro-defects to cause cleavage fracture. An historical review of these aspects can be found in [3].

At the macroscopic scale, local approach to fracture [4], and global approach [5] rely on the interpretation of micromecha- 
nisms of fracture effects on macroscopic fracture behavior, with good results [4], but also some limitations. These limitations can be considered as inherent to phenomenological fitting procedures [6]. Numerous works improved the results of this approach, but no real improvement have been done to link micromechanisms of fracture and macroscopic properties since Beremin's workgroup pioneering work.

This point was already emphasized in previous work [7]. Another aspect was highlighted : when the statistical aspect of cleavage fracture is to be explained, one generally takes into account the statistical aspect of the defects size repartition considered as cleavage triggering sources $[8,9]$. On the other side, models also generally rely on a microscopically uniform stress repartition, which is not fully satisfying. Heterogeneities in stress repartition at the microscopic scale might be a source of statistical variation. Heterogeneities of mechanical fields at the microscopical scale can be described conveniently by different approaches. MF models $[10,11]$ are generally used as way to consider heterogeneities at the interphase or intergranular scale. Recent progress in computational power allows to see FE analysis of microplasticity in 3D polycrystalline aggregates as a promising tool to investigate the intergranular but also the intragranular heterogeneities [12,13].

The present work will focus on a precise experimental description of stress repartition in 16MND5 french RPV steel during plastic straining at low temperature. Effects of microstructural heterogeneities are discussed. Stress heterogeneities are determined using X-Ray Diffraction methods (XRD).

Micromechanical modeling involves a crystal plasticity model and takes into account carbides clusters effect using a MF approach. The resulting behavior is then introduced in realistic 3D polycrystalline aggregates computed by FE methods. Calibration of the model parameters is made on macroscopic tensile testing. Validity of the modeling is then checked at the interphase and intergranular scales thanks to XRD results.

This modeling is then used on a smaller but more accurately described polycrystalline RVE, from which an EV consistent with the one used in local approaches to fracture is extracted. Different stress triaxialities are used for straining of this $\mathrm{EV}$ at $-150^{\circ} \mathrm{C}$, and a "Griffith-like" stress criterion for fracture, applied on crystallographic cleavage planes is used to compute time to fracture for different micro-cracks random distributions based on experimental distributions. Equivalent $\sigma_{u}$ and $m$ parameters are determined for different stress triaxialities. Effect of stress triaxiality on these parameters is eventually discussed as a matter of effective cleavage stress distribution on crystallographic cleavage planes inside the EV.

Previous work presented in [7] discussed a similar posttreatment of tensile simulations of multicrystalline aggregates, considering the ferritic packet as an elementary volume for cleavage triggering. Weaknesses of this modeling were identified as (i) carbide clusters role in plasticity mechanisms was not taken
Table 1. CHEMICAL COMPOSITION OF 16MND5 STEEL (weight-\%)

\begin{tabular}{llllllll}
\hline $\mathrm{C}$ & $\mathrm{S}$ & $\mathrm{P}$ & $\mathrm{Mn}$ & $\mathrm{Si}$ & $\mathrm{Ni}$ & $\mathrm{Cr}$ & $\mathrm{Mo}$ \\
\hline 0.159 & 0.008 & 0.005 & 1.37 & 0.24 & 0.7 & 0.17 & 0.5 \\
\hline \hline $\mathrm{V}$ & $\mathrm{Cu}$ & $\mathrm{Co}$ & $\mathrm{Al}$ & $\mathrm{N}$ & $\mathrm{O}$ & $\mathrm{Sn}$ & $\mathrm{As}$ \\
\hline$<0.01$ & 0.06 & $<0.01$ & 0.023 & 0.07 & $35 \mathrm{ppm}$ & $50 \mathrm{ppm}$ & $180 \mathrm{ppm}$ \\
\hline
\end{tabular}
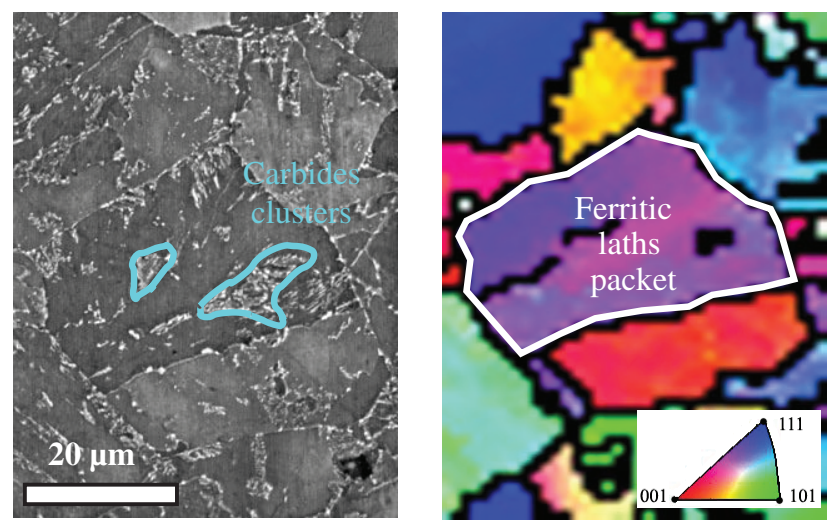

Figure 1. 16MND5 MICROSTRUCTURE CROSS-OBSERVATION BY SEM AND EBSD.

into account, (ii) taking average of stresses inside ferritic packets as a criterion leads to cleavage propagation probability underestimation and (iii) considered tensile stress state does not represent realistic loading for cleavage. These issues are addressed in this paper.

\section{MATERIAL CHARACTERIZATION Metallographic analysis.}

The main goal of this section will be to link the microstructural entities repartition with mechanical fields heterogeneities at the microscopic scale. This correct description is essential for further correct multiscale modeling of the material.

16MND5 bainitic steel is a low-alloyed steel commonly used for french RPV construction. Its chemical composition is reported in table 1 . The material undergoes several thermal treatments during its elaboration (i.e. forging, austenitization, water quenching and normalization) that leads to a so-called "tempered bainite" structure [14]. During the process, ferritic laths packets grow inside former austenitic grains. A packet can be defined by EBSD analysis as a group of laths slightly disoriented between each others [15]. The low carbon solubility of ferrite BCC lattice tends to create carbides inside ferritic packets and at their boundaries. In this particular steel, one can notice that carbides 
can grow close to each others, forming carbide clusters. Figure 1 illustrates these aspects, and highlights these entities. Thus, this particular bainitic microstructure will now be considered as a ferritic matrix distributed in lath packets of equivalent crystallographic orientation, and reinforced with carbide clusters.

\section{Heterogeneities observation using XRD at $-150^{\circ} \mathrm{C}$}

Composite effect A specific micro-tensile device with regulated cooling facility was previously developed [16] and used during this work. Specimen preparation details can be found in [3].

The main constituents of 16MND5 steel, ferrite and cementite carbides, are known for their difference in yield strength. Carbides have quite a higher strength that ferrite. Thus, during plastic straining of the material, carbides support a higher stress than ferrite, which accommodates the imposed strain and supports plasticity. Another convenient way to quantify these heterogeneities is the use of XRD method. Ferrite is quite a good candidate for XRD using $K \alpha$ emission of a chromium anti-cathod diffraction on ferrite's $\{112\}$ plane family. One can find thorough explanation about elastic strain measurement using XRD in [17]. A summary of these aspects leads to the fact that XRD allow measurement of the elastic strain $\varepsilon_{\phi \psi}$ inside the diffracting grains of the measuring spot for an incident beam orientation characterized by angles $\phi$ and $\psi$. These angles are expressed in the sample coordinate system on figure 2. Elastic strain is deduced from the variation of distance between crystallographic planes of the studied diffracting family.

Associated with the so-called " $\sin ^{2}(\psi)$ " method, XRD allows for determining stress $\sigma_{\phi}$ inside the studied constituent in a determined direction (i.e. for a determined $\phi$ ). This stress is directly related to the slope of the curve $\left(\varepsilon_{\phi \psi}, \sin ^{2} \psi\right)$ drawn for different $\psi$ values, with respect to the generalized Hooke's elasticity law expressed in the XRD coordinates system :

$$
\varepsilon_{\phi \psi}=\frac{1}{2} S_{2}\left(\sigma_{\phi}\right) \cdot \sin ^{2} \psi+S_{1} \cdot \operatorname{tr}(\underset{\sigma}{)})
$$

This simplified expression is only valid assuming that the

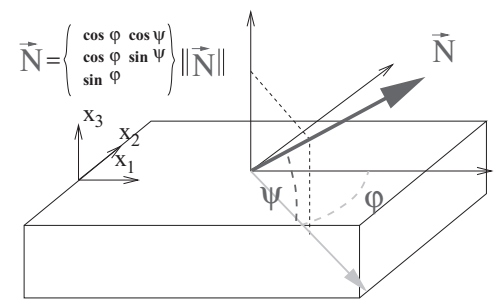

Figure 2. XRD COORDINATE SYSTEM stress $\sigma_{33}$ normal to the sample and the shearing stress in $\phi$ direction inside the irradiated volume are negligible. $S_{1}$ and $S_{2}$ are XRD elastic constants, and were deduced from mechanical elastic constants. If the $\phi$ direction is chosen as the tensile direction $(\phi=0)$, one can determine the average stress $\varepsilon_{11}^{\text {ferrite }}$ inside the ferritic matrix. Associated to the macroscopic stress indication given by the tensile device, stress repartition between phases can be observed. These aspects are illustrated in figure 3.a and 3.b. All XRD experiments were conducted during in-situ tensile testing with sample at $-150^{\circ}$ Celsius. After unloading, the difference between stresses in the bainite and the ferritic phase (approximately $150 \mathrm{MPa}$ ) remains, since they will both elastically unload and share really close Young moduli. This can be observed on the figure 3.b. The $\left(\varepsilon_{\phi \psi}, \sin ^{2} \psi\right)$ curves used for stress determinations have quite pronounced undulations around the mean slope line. This is an effect of polycrystalline heterogeneous straining which will be discussed further.

Polycrystalline effect Aside from the composite effect, differences in ferrite's BCC matrix orientations between lath packets is another source of mechanical heterogeneities. Its effect is quite comparable to what is known inside polycrystalline "grain-structured" materials [1]. During in-situ tensile testing inside a SEM, one can observe high strain heterogeneities on the surface relief, particularly at the lath packets boundaries, which allows qualitative observation of misoriented packets strain incompatibilities [3].

Strain heterogeneities will have an effect on the undulation around the mean slope line of the $\left(\varepsilon_{\phi \psi}, \sin ^{2} \psi\right)$ curve. They can be observed in the figure 3.c. These undulations are not relevant during loading, since curve's high mean slope value (related to high tensile stress) hides undulations effect, but after unloading, a relatively low mean residual stress state allows observing them.

Undulations are usually associated with crystallographic texture of the polycrystalline material. This is not the case here since no texture was present in the strain-free material [3] and no strong texture can develop with such small tensile straining (until $15 \%)$. We assume they are related to the fact that the diffracting volume (i.e. irradiated packets which have one $\{112\}$ family plane suited for diffraction) will be different for each $\psi$ measurement. This implies a difference in BCC lattice orientation, and thus a variation in local yield stress and hardening. This effect is illustrated on figure 3.d. Undulations are also aggravated because ferrite is hereby strained (i) in presence of carbides which increases the equivalent stress in ferrite and (ii) at low temperature which causes the yield strength to rise. Thus, undulations can be seen as a "snapshot" of the stress heterogeneities inside the ferritic matrix after unloading. These experimental results provide an original tool to observe stress heterogeneities and will be used to validate the multiscale modeling results. 


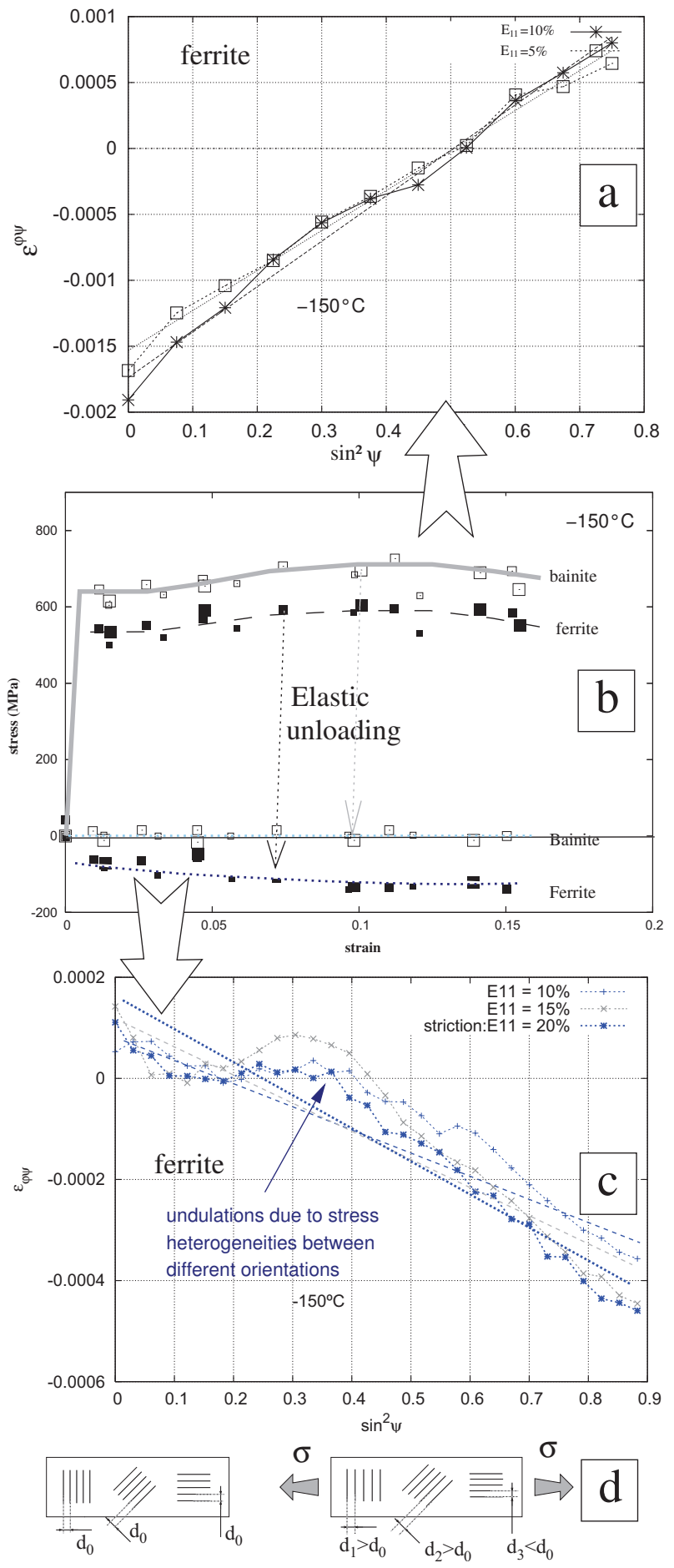

Figure 3. STRESS STRAIN MEASUREMENTS AT $-150^{\circ} \mathrm{C}$ FOR BAINITE AND FERRITE. (a) $\left(\varepsilon_{\phi \psi}, \sin ^{2} \psi\right)$ CURVES DURING TENSILE TEST. (b) STRESS STRAIN CURVES. (c) $\left(\varepsilon_{\phi \psi}, \sin ^{2} \psi\right)$ CURVES AFTER UNLOADING. (d) ELASTIC STRAIN HETEROGENEITIES.
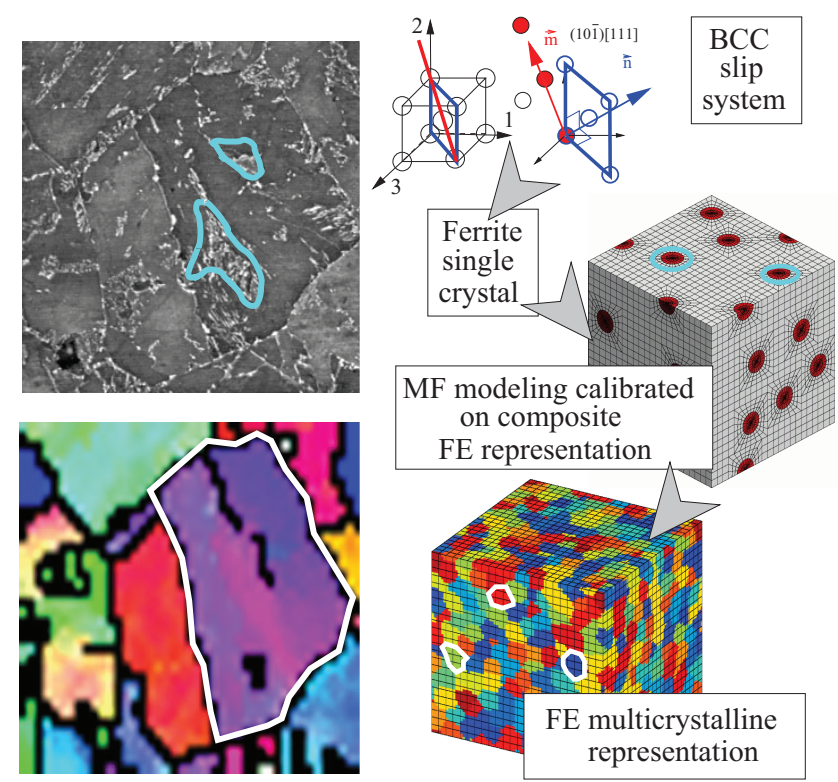

Figure 4. ILLUSTRATION OF THE MULTISCALE APPROACH USED FOR 16MND5 MODELING.

\section{MULTISCALE MODELING OF 16MND5 BEHAVIOR Modeling strategy}

Modeling will be mainly constrained by representation scales furtherly considered. Since microscopic local modeling of fracture is to be considered, the lower scale representation will be a micromechanical elastoplastic representation of ferritic phase behavior. Then, composite effect will be taken into account thanks to a MF model, and the resulting behavior will be implemented directly in the FE representation of a multicrystalline aggregate [12]. This approach implies scale transitions illustrated in figure 4.

Ferrite single crystal representation The constitutive equations of ferrite behavior modeling can be found in equation 2.

\begin{tabular}{|c|c|}
\hline total strain & $\underset{\sim}{\varepsilon}={\underset{\sim}{\varepsilon}}^{e}+{\underset{\sim}{\varepsilon}}^{p}$ \\
\hline stress & $\underset{\sim}{\sigma}=\underset{\approx}{\mathbf{C}}:{\underset{\sim}{\varepsilon^{e}}}^{e}$ \\
\hline plastic strain & ${\underset{\varepsilon}{\varepsilon^{p}}}^{p}=\sum_{g=1}^{24} \gamma^{g} \stackrel{\mathbf{R}}{g}^{g}$ \\
\hline projector & ${\underset{\sim}{\sim}}^{g}=1 / 2\left(\underline{\mathbf{m}}^{g} \otimes \underline{\mathbf{n}}^{g}+\underline{\mathbf{n}}^{g} \otimes \underline{\mathbf{m}}^{g}\right)$ \\
\hline shear strain rate & $\dot{\gamma}^{g}=\left(\frac{\left|\tau^{g}\right|-\tau_{c}^{g}}{K}\right)^{n} \cdot \operatorname{sign}\left(\tau^{g}\right)$ \\
\hline hardening & $\tau_{c}^{g}=\tau_{c_{0}}^{g}+Q^{g} \sum_{h=1}^{24} h^{g h} \cdot\left(1-e^{-b^{g} \cdot \gamma_{c u m}{ }^{h}}\right)$ \\
\hline
\end{tabular}

The ferrite elastoplastic strain $\underset{\sim}{\varepsilon}$ will be expressed as the sum 


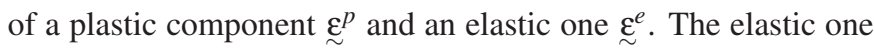
follows generalized Hooke's law within small perturbation assumption ( $\underset{\approx}{\mathbf{C}}$ representing isotropic elastic moduli tensor).

Plastic gliding in iron BCC lattice is considered on two slip systems families, $<111>\{110\}$ and $<111>\{112\}$. The number of crystallographic planes of the 110 and 112 families combined with the cubic symmetry of lattice enables 24 gliding systems.

The plastic strain rate tensor $\underset{\dot{\varepsilon}}{\dot{\varepsilon}^{p}}$ is then the sum of the shear strain rates $\dot{\gamma}^{g}$ over all the activated slip systems $g . \mathbf{R}^{g}$ is a geometrical projection tensor, depending of the normal to the slip system $\underline{\mathbf{n}}^{g}$ and the direction of the slip system $\underline{\mathbf{m}}^{g}$. These vectors are represented on figure 4

$\dot{\gamma}^{g}$ depends on $\tau^{g}$, the Resolved Shear Stress (RSS), the projection of the stress tensor $\sigma$ on the considered slip system $g$, and

of the actual critical RSS $\tau_{c}{ }^{g}$ [18]. $K$ and $n$ are parameters that have to be calibrated depending on the needed behavior.

$\tau_{c}^{g}$ evolution is described by hardening relations, $\tau_{c_{0}}^{g}$ being the initial critical RSS on the considered system $g$, which can be seen as the limit for micro-plasticity triggering. $Q^{g}$ and $b^{g}$ are parameters of the isotropic hardening formulation and $h^{g h}$ is the hardening matrix, describing interaction between slip systems (i.e. relative hardening effect of system $h$ on system $g$ ). One should notice that this formulation is considered as elastoviscoplastic, but a wise choice of parameters $K$ and $n$ annihilates viscous effect, leading to elastoplastic behavior within standard strain rates $\left(\varepsilon \in\left[10^{-6} ; 1\right]\right.$.

Composite effect representation Previous work put in evidence the fact that carbides clusters have a different effect on local behavior than the effect of homogeneously distributed carbides [3]. In order to properly take this effect into account, the second phase in the composite representation will be "carbide clusters", which involves carbides, but also the ferrite surrounding them. This was illustrated on the figure 4 : the line that surrounds carbides represents what will be now indicated as a "carbides cluster". As a simplification, these clusters will be considered as spheric in our representation.

This representation will imply a MF model, including "ferrite" phase and "carbides clusters" one. Ferrite will be represented using the micromechanical behavior described above, and carbide clusters will be considered as elastic. Elastic moduli used for clusters are the same as ferrite ones. The chosen MF modeling is the so-called " $\beta$ model" [19]. This model allows to consider the behavior of a RVE (i.e. the relation between global stress and strain, $\underset{\sim}{R V E}$ and ${\underset{\sim}{\mathbf{E}}}^{R V E}$ ), as a function of phases behaviors (relation between each phase $n$ stress and strain, $\sigma^{n}$ and $\underset{\sim}{\mathcal{q}^{n}}$ ). In our case spherical morphology of clusters and isotropic formulation of elasticity for both phases of composite conducts to the simplified formulation of the model summarized in 3 .

\begin{tabular}{|c|c|}
\hline Hill-Mandel Lemma & and $\left\{\begin{array}{l}\underset{\sim}{\mathbf{E}}=<\underset{\sim}{\boldsymbol{\varepsilon}}>_{R V E}=<{\underset{\sim}{\mathbb{V}^{n}}}^{n}>_{R V E} \\
\underset{\sim}{\mathbf{S}}=<\underset{\sim}{\mathbf{S}}>_{R V E}=<{\underset{\sim}{\sigma^{n}}>_{R V E}}\end{array}\right.$ \\
\hline localization & ${\underset{\sim}{\sigma}}^{n}=\underset{\sim}{\mathbf{S}}+2 \mu(1-\beta)\left(\underset{\sim}{\mathbf{B}}-\beta^{n}\right)$ \\
\hline phase hardening & 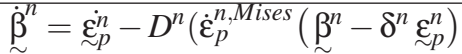 \\
\hline mean field & $\underset{\sim}{\mathbf{B}}=\left\langle\underline{\sim}^{n}\right\rangle_{R V E}$ \\
\hline isotropic elasticity & $\beta=\frac{2(4-5 v)}{15(1-v)}$ \\
\hline von Mises equ. strain & $\varepsilon^{\text {Mises }}=\sqrt{\frac{2}{3} \underset{\sim}{\underset{\sim}{\varepsilon}: \underset{\sim}{\varepsilon}}}$ \\
\hline
\end{tabular}

A localization rule links phase $n$ stress to the global stress in RVE and phases strains. $\mu$ and $v$ are respectively the shearing elastic modulus and the Poisson ration. This modeling has the particularity to allow a phenomenological description of the interphase hardening, using the $\delta^{n}$ and $D^{n}$ coefficients. This ability was found convenient in this case compared to classical MF models. These coefficients have to be tuned on an explicit morphological representation of the composite. This calibration was made on a representative FE composite morphology illustrated in figure 4, using same phases behavior for clusters and ferrite in MF and FE models. Carbides clusters behavior description will lack $\delta$ and $D$ coefficients : as elastic phases, no interphase hardening will be described.

Multicrystalline representation The presented MF model is then implemented at each Integration Point (IP) on a regularly meshed $3 \mathrm{D}$ aggregate, using Euler angles convention to represent BCC lattice orientation inside each lath packet. This simulated volume represents the final RVE. The orientation of laths packets were randomly affected in order to represent material isotropic crystallographic texture. Stress and strain fields are computed at each IP with a classical FE method. RVE used for model calibration is composed of 1000 packets, and is loaded in uniaxial tension.

\section{Results and validation}

Calibration results. One can conceive that the multiple scales involved in modeling results in a quite complex parameters calibration procedure. This procedure and the resulting calibrated parameters and coefficients are widely discussed in complement of relevant issues about modeling in [3]. The simulated global response of computed FE aggregates compared to the experimental one is presented in figure 5.

Stress and strain heterogeneities in bainite are also represented between aggregate's IP. One should remember that these results are only "the hidden part of the iceberg". Multiscale modeling allows much more extensive study of mechanical fields 
repartition inside aggregate, phases and inside ferritic phase, at the crystallographic scale.

Comparison with XRD results. As an example, the elastic strain inside ferritic phase can be easily extracted at each IP. A specific post-treatment was developed to simulate XRD diffraction results [1], which is made possible by the knowledge of attributed lattice orientations of ferrite.

A specific loading was applied to aggregates to take free surface into account, and a post-treatment was applied considering only the lath packets layer near the free surface. These consideration are linked to the fact that XRD involves a small layer in metallic materials (typically $20 \mu \mathrm{m}$ for ferrite, a value close to lath packet size), which is also a free surface during tensile test. Tensile straining of the aggregate was simulated until a macroscopic strain of $15 \%$ and followed by elastic unloading. These steps were essential to catch correct representation of stress heterogeneities involved in undulations of $\left(\varepsilon_{\phi \psi}, \sin ^{2} \psi\right)$ curves. A comparison between computed and experimental $\left(\varepsilon_{\phi \psi}, \sin ^{2} \psi\right)$ curves is made in figure 6 . The quite good agreement between simulation and experimental work is a consequence of choices made during the modeling part of this work.

One should notice that small perturbation assumption prevents any evolution of crystallographic texture during simulation (no lattice rotation). However, undulations are correctly represented. This validates the previously made hypothesis that they represent plastic heterogeneities between differently oriented packets. It is also worth noting that calibration process is made at the macroscopic scale, but is validated at lower scale without further calibration which gives a relative confidence in

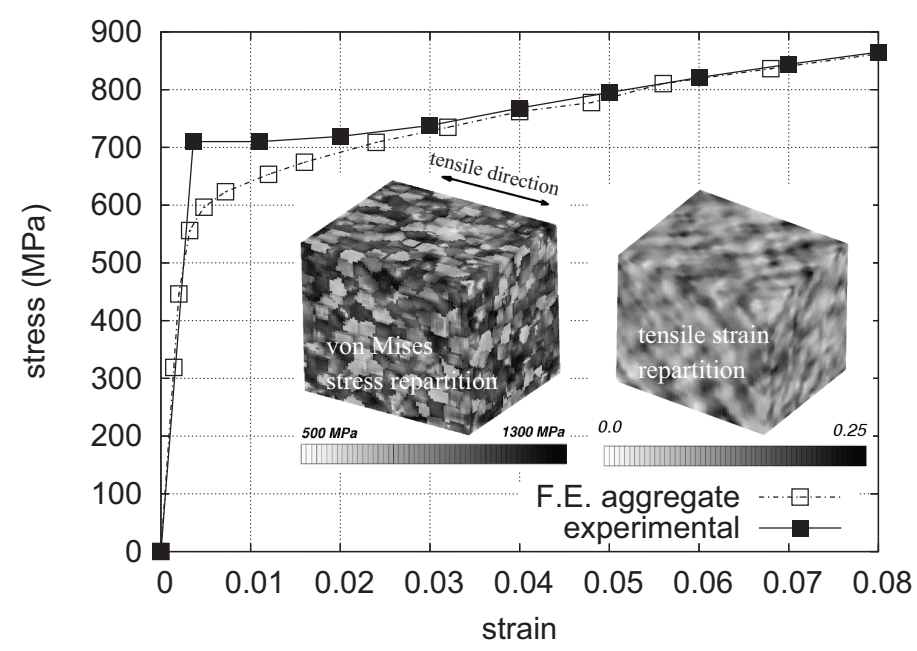

Figure 5. GLOBAL SIMULATED MACROSCOPIC RESPONSE. REPARTITION OF STRESS AND STRAIN IS REPRESENTED FOR A MACROSCOPIC TENSILE STRAIN OF $12 \%$.

This paper is posted here by permission of ASME for personal use only. Additional reproduction, redistribution, or transmission in either print or digital form is not permitted without ASMEs prior written permission.

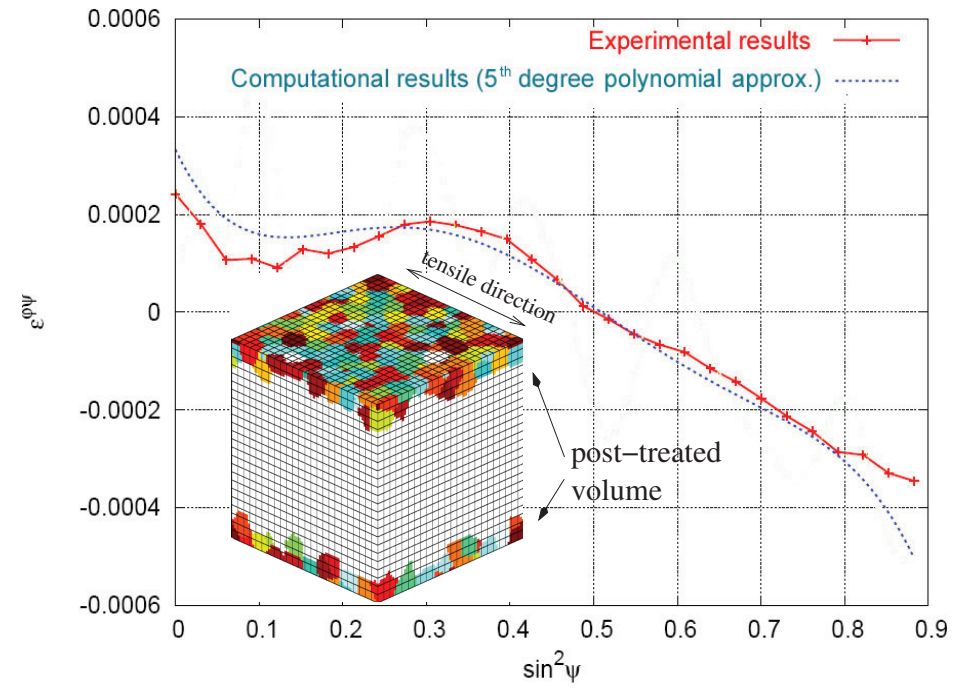

Figure 6. SIMULATED XRD $\left(\varepsilon_{\phi \psi}, \sin ^{2} \psi\right)$ UNDULATIONS AFTER UNLOADING FOR A MACROSCOPIC TENSILE STRAIN OF $15 \%$ at $-150^{\circ} \mathrm{C}$

the ferrite's stress representation.

\section{MICROSCALE MODELING OF BRITTLE FRACTURE}

On the basis of this fine micromechanical behavior description, a local fracture criterion will be applied at the microscopic scale (i.e. at every IP). This will be a deterministic process. Whereas RVE used for behavior identification needed to include a large amount of packets (1000, considered as an appropriate amount for a good behavior identification [12]), this one will have a more precise representation of stresses inside packets (i.e. more IP are used to represent a packet). Simulations made in this part of the work will thus imply a smaller RVE $\left(50 \times 50 \times 50 \times \mu m^{3}\right)$, that will also have the same size than the EV used in local approach of Beremin's workgroup [4].

\section{Cleavage modeling assumptions}

The hypothesis about cleavage used in this work are thoroughly discussed in [3]. They are mainly based on Beremin's workgroup assumptions, and adapted to more recent experimental observations. These hypothesis are listed below, and linked to post treatment equations proposed further :

1. Carbides are the initiation sites for microcracks. An experimental carbides sizes distribution will be considered (equation. 4.A).

2. The carbide population is divided into a population of small carbides gathered in clusters that was taken into account during the behavior modeling, but also into a population of bigger lonely carbides eligible for plastic crack initiation. Thus

\section{Copyright (c) 2008 by ASME}


a global carbides sizes distribution can be used, the distinction between the two populations roles being a natural process.

3. Once a defect is initiated, propagation in the surrounding ferritic matrix is a "Griffith-like" process. Local stresses on cleavage planes (4.B and 4.C) will be compared to a local Griffith propagation criterion using carbides sizes (equation. 4.D and 4.E).

4. The biggest carbides inside the microstructure are place of crack initiation during the earlier stage of plasticity. These cracks are quickly blunted in a similar manner as described by Haušild for manganese sulfides blunting [20,21], and can be eliminated of a micromechanical fracture process (equation. 4.D)

5. Initiation of micro-cracks requires plastic activity whether Smith's dislocation pile-up mechanism [22] or carbide cracking [20] is considered (equation. 4.F).

6. Microstructural barriers (i.e. disoriented lath packets boundaries crossing) are not relevant during 16MND5 brittle fracture [3]. The propagation of a micro-crack is a "weakest link"-type event [3].

\begin{tabular}{|c|c|}
\hline A carbide sizes & $p\left(r \geq r_{0}\right)=1-\exp \left(-\left(\frac{r_{0}}{3.6 .10^{-8}}\right)^{-2.7}\right)$ \\
\hline B projected stress & $\sigma_{f e r}^{p}=\underline{n}^{p} \cdot \underline{\sigma}_{f e r} \cdot \underline{n}^{p}$ \\
\hline C effective stress & $\sigma_{c, i}^{M a x}=\max \left(\sigma_{\text {fer }}^{100} ; \sigma_{\text {fer }}^{010} ; \sigma_{\text {fer }}^{001}\right)_{i}$ \\
\hline D propagation & $\sigma_{n, i}^{p}=\left\{\begin{array}{cll}\sqrt{\frac{\pi E \gamma_{R}}{2\left(1-v^{2}\right) r_{n, i}}} & \text { if } & r_{n, i} \leq r_{\max } \\
\sigma^{N P} & \text { if } & r_{n, i}>r_{\max }\end{array}\right.$ \\
\hline E Griffith stress & $\sigma_{i}^{\text {grif }}=\left\{\begin{array}{cll}\min \left(\sigma_{n, i}^{p}\right) & \text { if } & n_{i}>0 \\
\sigma^{N P} & \text { if } & n_{i}=0\end{array}\right.$ \\
\hline F stress triaxiality & $\chi=\frac{1+2 \alpha}{3(1-\alpha)} \quad$ with $\quad S_{22}=S_{33}=\alpha S_{11}$ \\
\hline G cleavage triggering & and $\left\{\begin{array}{l}\sigma_{c, i}^{\text {max }} \geq \sigma_{i}^{\text {grif }} \\
\varepsilon_{p, i}^{e q u} \geq \varepsilon_{p}^{\text {mini }}\end{array}\right.$ \\
\hline
\end{tabular}

Carbides population, cleavage criterion The experimental repartition of carbides sizes used in this work was found in [23]. It was extracted from the German Euro "A" steel study, a material similar to the french 16MND5. It is represented in figure 7 .a.

This statistical repartition comes with a precise carbide volumic counting $\left(N=7.6 \pm 2.4 \cdot 10^{17} \mathrm{~m}^{-3}\right)$, which lacks in previous works about carbides in A508 Cl.3 steel [8]. Otherwise, statistical carbide repartition reported in 16MND and Euro "A" are almost identical. In this count, smallest carbides under $10 \mathrm{~nm}$ diameter were ignored, which is not considered as an issue regarding hypothesis 3 . Knowing the EV size $V_{0}$, the number of IP in this volume $(18 \times 18 \times 18$ elements with $27 \mathrm{IP})$, this repar-

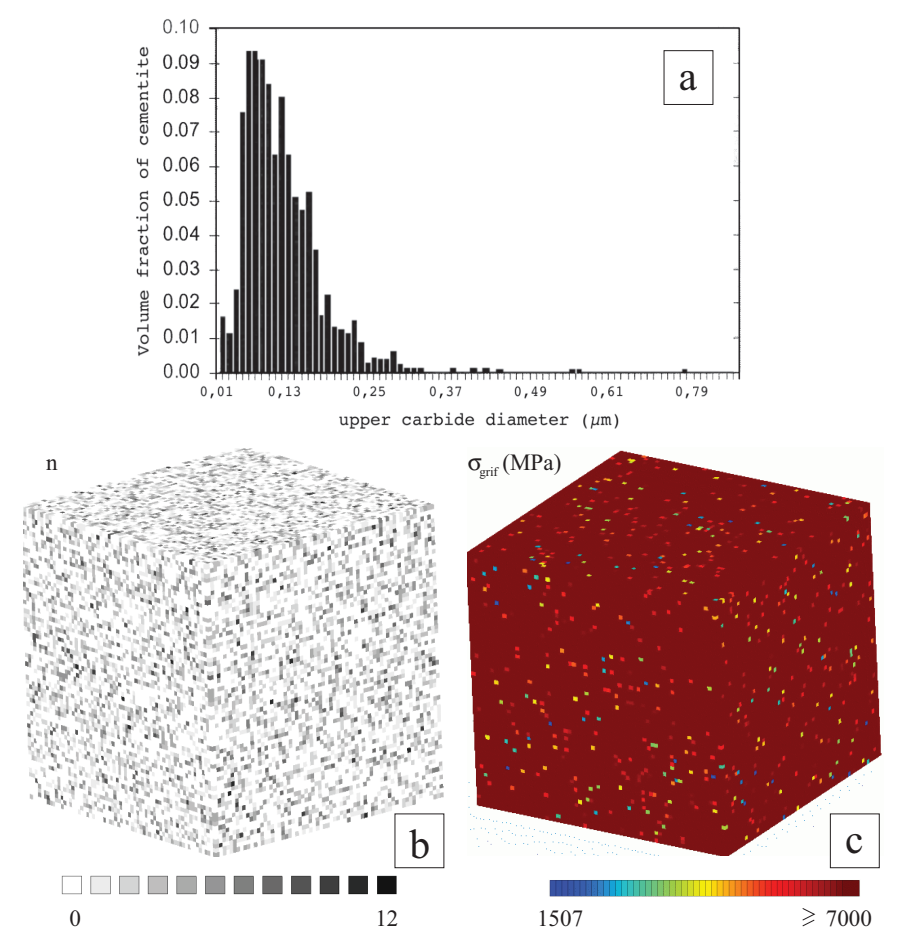

Figure 7. CARBIDES EXPERIMENTAL DISTRIBUTION [23], REPARTITION IN EV, AND RESULTING GRIFFITH STRESS REPARTITION.

tition can be used to attribute an average number of carbides $\bar{n}$ at each IP of 0.603 .

A truncated gaussian distribution of integers $(\bar{n}$, standard deviation $\omega_{n}=4$, truncated below 0 ) is used to attribute a carbide number $n_{i}$ at each integration point $i$. One can observe such a distribution in figure 7.b. Randomly attributed sizes $r_{n, i}$ are then attributed to these $n_{i}$ carbides using the repartition given in equation 4.A.

The propagation stress $\sigma_{n, i}^{p}$ is then deduced according to Griffith's criteria of propagation of a penny-shaped crack [24]. This propagation stress will be chosen as an arbitrary unreachable "non-propagation" stress value $\sigma^{N P}$ for carbides sizes upon $r_{\max }(1 \mathrm{~m})$, with respect to hypothesis 4 . This follows equation 4.D. $E$ represents the Young elastic modulus, and $\gamma_{R}$ represents the effective surface energy (i.e. ferrite's surface energy at $0^{\circ} \mathrm{K}$ plus energy involved in plastic dissipative mechanisms). It is worth noting that $\gamma_{R}$ value is discussed in [3]. The value used here, $\left(\gamma_{R}=6,78 \mathrm{~J} . \mathrm{m}^{-2}\right)$ comes from Wallin's hypothesis [5], of dependency of $\gamma_{R}$ with temperature applied to 16MND5 ferritic phase [3].

The Griffith critical stress $\sigma^{\text {grif }}$ at an IP $i$ will be the smallest value of $\sigma_{n, i}^{p}$, and will be taken as $\sigma^{N P}$ when no carbides were attributed as described in equation 4.E. Such a repartition of critical stress inside the RVE is represented in figure 7.c. 


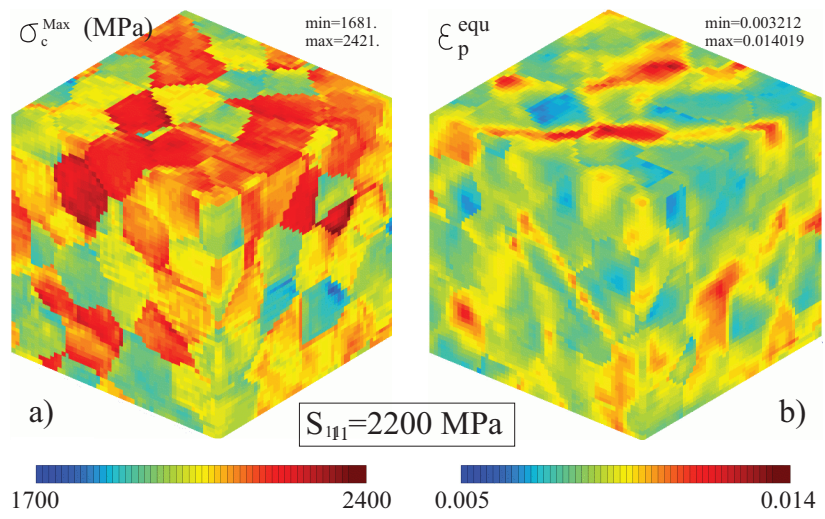

Figure 8. MAXIMUM CLEAVAGE STRESS AND EQUIVALENT PLASTIC STRAIN IN FERRITE. $T=-150^{\circ} \mathrm{C}, \chi=3$.

Triaxial stress state loading on RVE The stress inside ferritic phase can be projected onto $\{100\}$ family plane $p$, as presented in equation 4.B. The $\{100\}$ plane easilythat may involve cleavage at a given IP can reasonably be considered as the one between (100), (010) and (001) submitted to the highest stress, which gives sense to an effective cleavage stress $\sigma_{c, i}^{\text {Max }}$ (equation 4.C).

Cleavage initiation of steels involves high local stress values, that cannot be accessed using uniaxial loadings on RVE. Thus a triaxial stress state was applied. This was obtained applying a purely hydrostatical stress tensor $\underset{\sim}{\mathbf{S}}$ on the RVE. $\chi$ representing stress triaxiality (equation 4.F). It is worth noting that $\mathbf{\sim}$ diagonal components can also be taken as its principal values in this setting $\left(\Sigma_{1}=S_{11}\right)$, and that the stress triaxiality remains a constant during loading of the RVE. Boundary conditions issues were cautiously avoided. This was done by performing simulations on a larger volume, and extracting only the central zone of interest $V_{0}$.

Figure 8 shows the maximum cleavage stress $\sigma_{c}^{\operatorname{Max}}$ and equivalent plastic strain $\varepsilon_{p}^{\text {Mises }}$ in ferrite for this RVE loaded with a triaxial stress state.

Link with local approach Random distribution of carbides will be computed $X$ times, and for each computation $x$, the time to fracture is estimated as the time where terms of equation 4.G are fullfilled onto at least one IP $\left(\varepsilon_{p}^{\min i}=10^{-5}\right)$. This was made according to the weakest link assumption in hypothesis 6 . A linear interpolation was used for time and first principal stress value estimation between simulated time steps.

This leads to $X$ values of first principal stress to fracture. They were sorted in increasing order (i.e. $x=1$ for the smallest $\Sigma_{1}$ value). A cumulative probability of fracture was plotted as a function of $\Sigma_{1}$ stress to fracture using the estimator $P_{f}=(x-$ $0.5) / X$. The shapes of the curves, presented in figure 9.a, recalls the Beremin's failure cumulative probability evolution shape :

$$
P_{f}=1-\exp \left(-\frac{\sigma_{w}}{\sigma_{u}}\right)^{m}
$$

$\sigma_{w}$ being the Weibull stress [4]. Cumulative fracture probabilities presented in this work were obtained with the same mechanichal field, but with different carbides distribution. It is thus equivalent to a cumulative probability that one would obtain considering a macroscopic structure composed of $X \mathrm{EV}$ identical to the RVE, homogeneously loaded with $\mathbf{S}$ stress state, were the carbide population can be described by equation 4.A. Assuming the whole volume undergoes plasticity [25], one can show that this is similar to the use of Beremin's approach considering a virtual macroscopic volume composed of $X \mathrm{EV}$.
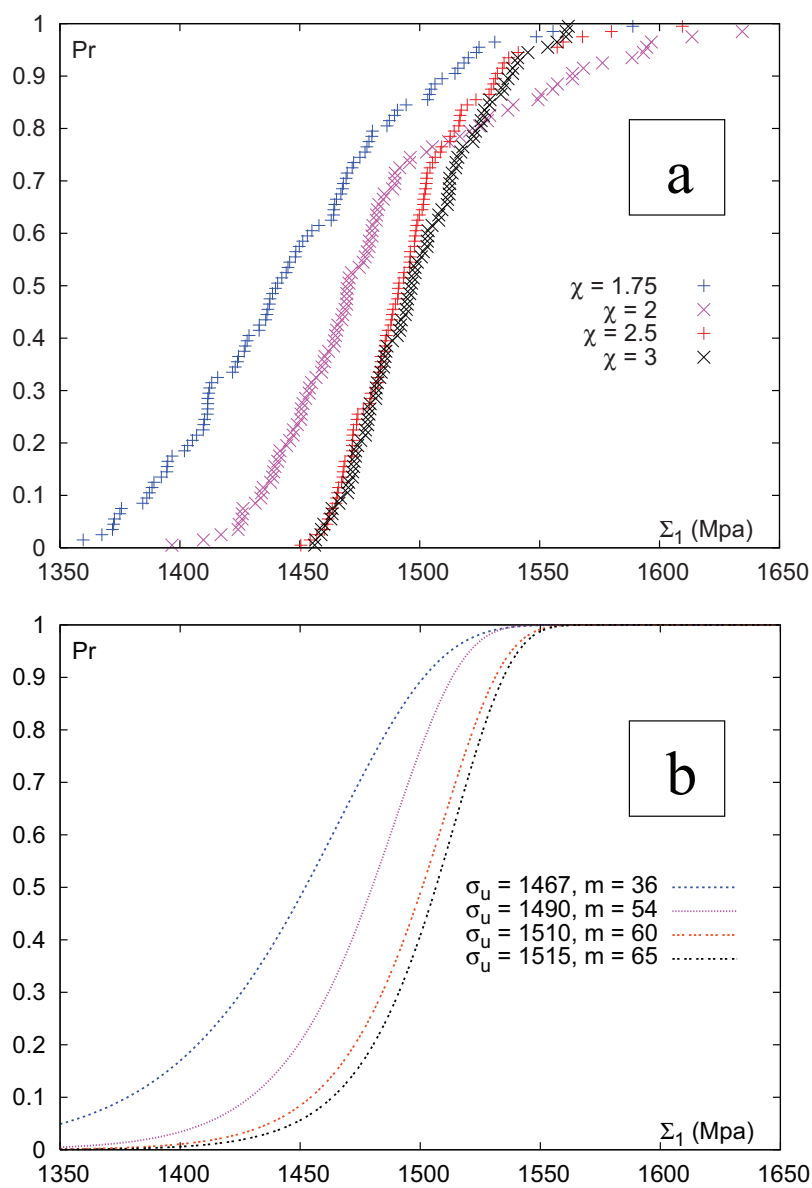

Figure 9. CUMULATIVE PROBABILITY OF FRACTURE FOR DIFFERENT TRIAXIALITIES. a) SIMULATED RESULTS b) Gnuplot FIT WITH $1-\exp \left(-\frac{\sigma_{w}}{\sigma_{u}}\right)^{m}$ CURVES

This paper is posted here by permission of ASME for personal use only. Additional reproduction, redistribution, or transmission in either print or digital form is not permitted without ASMEs prior written permission. 
Table 2. VALUES OF $m$ AND $\sigma_{u}$ FOR DIFFERENT TRIAXIALITIES $\chi$

\begin{tabular}{lllll}
\hline$\chi$ & 1.75 & 2 & 2.5 & 3 \\
\hline$\sigma_{u}(\mathrm{MPa})$ & 1467 & 1490 & 1510 & 1515 \\
\hline$m$ & 36 & 54 & 60 & 65 \\
\hline
\end{tabular}

Gnuplot was used to fit equation 5 to the plotted results, leading to values of $m$ and $\sigma_{u}$ reported in table 2 and figure 9.b.

\section{Discussion}

$m$ parameter tends to increase with stress triaxiality, whereas $\sigma_{u}$ values evolution with $\chi$ can be found astonishing : it tends to indicate that a higher stress triaxiality would be favorable to "cleavage resistance". These results have to be discussed as cross effects of $\sigma^{\text {grif }}$ (related to $\gamma_{R}$ ) and $\sigma_{c}^{\text {Max }}$.

The evolution of $m$ parameter appears simple to understand and to explain. $\sigma_{c}^{\operatorname{Max}}$ stress distribution inside the EV is plotted for a macroscopic stress really close to calculated values of $\sigma_{u}\left(\Sigma_{1}=1500 \mathrm{MPa}\right.$ see figure 10$)$. One can easily observe that the effective cleavage stress $\sigma_{c}^{\text {Max }}$ distribution is far more spread for low triaxialities. This makes the event of fracture (i.e. having a big carbide in an IP loaded with a high effective cleavage stress) less probable than, on the other side, with tight distribution of $\sigma_{c}^{M a x}$ for high triaxialities, which makes the event of fracture quite inescapable once a certain average stress is reached.

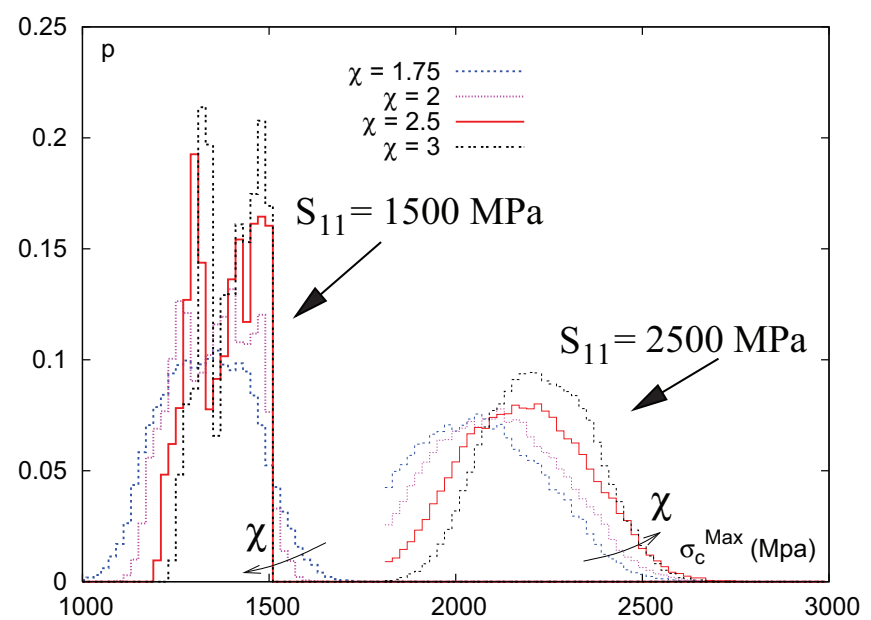

Figure 10. REPARTITIONS OF EFFECTIVE CLEAVAGE STRESS $\sigma_{c}^{M a x}$ INSIDE THE EV FOR VALUE OF $\Sigma_{1}$ OF $1500 \mathrm{MPa}$ (ACTUAL SITUATION) AND VALUE OF $\Sigma_{1}$ OF $2500 \mathrm{MPa}$ (VIRTUAL SITUATION). $\sigma_{u}$ evolution is less trivial. First, one should remark that $\sigma_{u}$ evolution with $\chi$ is not highly significant compared to $m$ evolution $\left(\left(\sigma_{u}^{\text {Max }}-\sigma_{u}^{\min }\right) / \sigma_{u}^{\text {Max }} \approx 3 \%\right.$ where $\left(m^{\text {Max }}-m^{\min }\right) / m^{\text {Max }} \approx$ $44 \%)$. Then, a cautious observation of figure 10 indicates that the tightening of $\sigma_{c}^{\text {Max }}$ distribution that explains $m$ increase comes with a retreat of the distribution tail. This can be related to the fact that plasticity, which is a great source of stress heterogeneity is far less developed for low triaxiality, and thus explains $\sigma_{u}$ decrease.

To illustrate this, one can observe the same $\sigma_{c}^{M a x}$ distribution, for a virtual material which much higher equivalent surface energy $\gamma_{R}$, but exactly the same behavior at the microscopic scale on figure 10. This would allow much more plasticity to take place before fracture. If average $\sigma_{u}$ of $2500 \mathrm{MPa}$ could be reached, one can easily see that the tendency on $\sigma_{u}$ would be inverted : plasticity would have occurred in all cases and $\sigma_{c}^{M a x}$ distribution tails would be ordered in a more "instinctive" way. On the other hand, the effect on $m$ value would keep the same interpretation : the tails of $\sigma_{c}^{M a x}$ distributions are still tightened.

These results show that the triaxiality has an effect on the parameter $m$, whereas no clear nor absolute effect can be seen on $\sigma_{u}$, when considering the simulated fracture mechanisms on the RVE. This observation could be an explanation of the fact that identified $m$ and $\sigma_{u}$ values are not simply transferable between different fracture testing sample geometries. The variation of identified values of $m$ is pointed out in other works [6].

It is well known that different geometries will lead to different triaxialities at the sites of cleavage triggering, which certainly drives to different stress repartitions. Considering our triggering micromechanism is adequate, this work demonstrates that the first principal value of stress could be balanced somehow by the stress triaxiality on the EV in order to address geometrical issues.

\section{CONCLUSION}

A fine representation of plasticity at the microstructural scale has been set up, involving different scales that were considerated as appropriate. Comparison with experimental characterization using XRD after strainting at $-150{ }^{\circ} \mathrm{C}$ shows that this representation intrinsically reproduces stress heterogeneities inside the ferritic matrix between different crystallographic orientation without further calibration.

Based on this representation, a simple "Griffith-like" criterion was used to compute time to fracture of a RVE loaded with different triaxial stress state evolutions, with realistic defects distributions, and for a volume consistant with the local approach EV. A cumulative probability of fracture for the RVE was then computed, which showed evidence of stress triaxiality effects on probability of fracture of an EV. These effects are related to difference in stress repartition on crystallographic cleavage planes for the same value of first principal stress on the EV. 


\section{ACKNOWLEDGMENT}

G. Cailletaud and J. Besson from Ecole des Mines de Paris are greatly acknowledged for furnishing ZeBuLoN FE code and support. R. Pesci, M. Wary, and D. Bouscaud from ENSAM de Metz are to be thanked about help on the experimental part. Dr. D. P. G. Lidbury, D. W. Beardsmore and S. Ortner supported this work by furnishing information about carbide sizes distribution. J.-P. Mathieu would like to thank his 3 co-authors for spending three great years doing research at their sides.

\section{REFERENCES}

[1] Mathieu, J.-P., Berveiller, S., Inal, K., and Diard, O., 2006. "Plastic heterogeneities characterisation in 16MND5 RPV steel by X-Ray diffraction, comparison with finite-element approach". In Proceedings of the 7th European Conference on Residual Stress, Trans Tech Publications, pp. 523-528.

[2] Zener, C., 1948. "Fracturing of Metals". 29 th National Metal Congress and Exposition, American Society for Metals, pp. 3-31.

[3] Mathieu, J.-P., 2006. "Analyse et modélisation micromécanique du comportement et de la rupture fragile de l'acier 16MND5 : Prise en compte des hétérogénéités microstructurales.". $\mathrm{PhD}$ thesis, Ecole Nationale Supérieure d'Arts et Métiers - avail. at http://pastel.paristech.org.

[4] Beremin, F., 1983. "A local criterion for cleavage fracture of a nuclear pressure vessel steel”. Metall. Trans. A, 14, pp. 22772287.

[5] Wallin, K., Saario, T., and Torronen, K., 1984. "Statistical model for carbide induced brittle fracture in steels". Met. Sci., 18, pp. 1316.

[6] Bordet, S., Kartensen, A., Knowles, D., and Wiesner, C., 2005. "A new statistical local criterion for cleavage fracture in steel. Part I : model presentation". Eng. Frac. Mech., 72, pp. 435-452.

[7] Mathieu, J.-P., Berveiller, S., Inal, K., and Diard, O., 2006. "Multiscale modelling of cleavage fracture at low temperatures : influence of heterogeneities at the granular scale". Fat. Fract. Eng. Mater. Struct., 29, pp. 725-737.

[8] Lee, S., Kim, S., Hwang, B., Lee, B., and Lee, C., 2002. "Effect of carbide distribution on the fracture toughness in the transition temperature region of an SA 508 steel". Acta Mater., 50, pp. 47554762.

[9] Tanguy, B., Besson, J., and Pineau, A., 2003. "Comment on Effect of carbide distribution on the fracture toughness in the transition temperature region of an SA 508 steel". Scripta Mater., 49, pp. 191-197.

[10] Eshelby, J., 1957. "The determination of the elastic field of an ellipsoidal inclusion and related problems". Proc. Roy. Soc. London A, 241, pp. 376-396.

[11] Berveiller, M., and Zaoui, A., 1979. "An extension of the selfconsistent scheme to plastically-flowing polycrystals". J. Mech. Phys. Solids, 26, pp. 325-344.

[12] Barbe, F., Decker, L., Jeulin, D., and Cailletaud, G., 2001. "Intergranular and intragranular behavior of polycrystalline aggregates. Part 1 : F.E. Model". Int. J. Plasticity, 17, pp. 513-536.

[13] Diard, O., Leclerq, S., Rousselier, G., and Cailletaud, G., 2005.
"Evaluation of finite element based analysis of 3D multicristalline aggregates plasticity - Application to crystal plasticity model identification and the study of stress and strain fields near grain boundaries". Int. J. Plasticity, 21, pp. 691-722.

[14] Bhadeshia, H., and Honeycombe, R., 2006. Steels - Microstructures and Properties. Butterworth-Heinemann.

[15] Obrtlik, K., Robertson, C., and Marini, B., 2006. "Dislocation structures in 16MND5 pressure vessel steel strained in uniaxial tension". J. of Nucl. Mat., 342, pp. 35-41.

[16] Pesci, R., Inal, K., Berveiller, S., Patoor, E., Lecomte, J.-S., and Eberhardt, A., 2006. "Inter and intragranular stress determination with kossel microdiffraction in a scanning electron microscope". In Proceedings of the 7th European Conference on Residual Stress, Trans Tech Publications, pp. 109-114.

[17] Hauk, V., 1997. Structural and residual stress analysis by nondestructive methods. Elsevier Science B.V., Amsterdam.

[18] Méric, L., and Cailletaud, G., 1991. "Single crystal modelling for Structural Calculations : Part 2 - F.E. Implementation”. J. Eng. Mat. Tech., 113, pp. 171-182.

[19] Cailletaud, G., and Pilvin, P., 1994. "Utilisation de modèles polycristallins pour le calcul par éléments finis". Rev. Européenne des Éléments Finis, 3, pp. 515-541.

[20] Ortner, S., 2002. "Factor affecting the shape of the ductile-tobrittle transition". Int. J. of Press. Vessels and Piping, 79, pp. 693700 .

[21] Hausild, P., 2002. "Transition ductile-fragile dans un acier faiblement allié". PhD thesis, Ecole Centrale de Paris.

[22] Smith, E., 1966. "The nucleation and growth of cleavage microcracks in mild-steels". Proceedings Conference on 'Physical basis of yield and fracture'. Institute of Physics and Physical Society, Oxford London, pp. 36-46.

[23] Ortner, S., Duff, J., and Beardsmore, D., 2005. "Characterisation of Euro A Reference steel for application of EOH model of brittle fracture - Technical report SA/EIG/15234/R003 - Project PERFECT". SERCO Assurance.

[24] Griffith, A., 1920. "The phenomena of rupture and flow in solids." Phil Trans. R. Soc. A, 221, pp. 163-198.

[25] Mathieu, J.-P., Berveiller, S., Inal, K., and Diard, O., 2006. "Computation of brittle fracture local probability of a RPV bainitic steel based on realistic microstructure representation". In Proceedings of the 9th European Mechanics of Materials Conference, Les Presses - Ecole des Mines, pp. 435-440. 